\title{
The count of tender rather than swollen joints correlates with aortic stiffness in patients with rheumatoid arthritis
}

\author{
Konstantinos Triantafyllias ${ }^{1 *}$, Michele De Blasi ${ }^{1}$, Isabell Hoffmann ${ }^{2}$, Thomas Thomaidis ${ }^{3}$, Philipp Drees ${ }^{4}$ \\ and Andreas Schwarting ${ }^{1,3}$
}

\begin{abstract}
Background: Patients with rheumatoid arthritis (RA) are at a higher cardiovascular (CV) risk in comparison to the general population. CV risk associates closely with aortic stiffness. Aim of this exploration was therefore to evaluate aortic stiffness in patients with RA and to examine its association with various RA associated parameters as well as with traditional CV risk factors.

Methods: Measurements of carotid-femoral pulse wave velocity (cfPWV) were analyzed retrospectively in 38 RA patients and 25 controls. We investigated the statistical difference between cfPWV values in the two groups. Furthermore, we analyzed the associations of cfPWV with laboratory and clinical RA parameters including Disease Activity Score 28 and its components, rheumatoid factor, cyclic citrullinated peptide antibodies, antinuclear antibodies and RA duration. Finally, we explored the relationship of cfPWV with traditional CV risk factors in the RA group.

Results: cfPWV was not significantly higher in RA patients in comparison to controls in an adjusted statistical model for confounding factors [ $-0.58795 \% \mathrm{Cl}(-1.38$ to 0.201$), p=0.144]$. Among RA patients there was a statistically significant correlation of cfPWV with age (rho $=0.544, p=0.001)$ and the count of tender joints $[0.05195 \% \mathrm{Cl}$ $(0.008-0.207), p=0.034]$. Finally, C-reactive protein associated only marginally with cfPWV $[0.10595 \% \mathrm{Cl}(-0.410$ to $0.003), p=0.053]$.
\end{abstract}

Conclusions: In RA patients the number of tender, rather than swollen joints correlates with stiffness of the aorta, as measured through cfPWV. Therefore, RA associated joint pain might play a role in the development of aortic stiffness and thus increase CV risk.

Keywords: Rheumatoid arthritis, Aortic stiffness, Joint tenderness, Pain, Cardiovascular risk, Carotid-femoral pulse wave velocity

\section{Background}

Autoimmune diseases, such as rheumatoid arthritis (RA), correlate with high cardiovascular $(\mathrm{CV})$ risk and high morbidity and mortality rates (Abou-Raya and AbouRaya 2006). In particular, CV diseases are considered to be responsible for the cause of $40-50 \%$ of all deaths in patients with rheumatoid arthritis (del Rincón et al.

\footnotetext{
*Correspondence: ktriantafyllias@gmail.com

${ }^{1}$ ACURA Rheumatology Clinics, Kaiser-Wilhelm-Str. 9-11,

55543 Bad Kreuznach, Germany

Full list of author information is available at the end of the article
}

2001). CV risk in the general population can be assessed through various markers, one of which is arterial stiffness. Particularly, stiffness of the aortic vasculature is a modifiable, independent predictor of $\mathrm{CV}$ risk and can be measured through carotid femoral pulse wave velocity (cfPWV) (Laurent et al. 2006). The predictive value of this marker concerning $\mathrm{CV}$ events has been shown in a series of epidemiological studies and cfPWV is nowadays characterized as the gold standard for the assessment of aortic stiffness (Laurent et al. 2006).

cfPWV gives information about the elasticity of the vascular bed among the carotid and the femoral artery

\section{Springer Open}


(Baulmann et al. 2010). In contrast to parameters such as blood pressure, lipids or glucose which match the instantaneous intensity of traditional CV risk factors, cfPWV reflects the long-term effects of established and unknown risk factors together with the individual genetic predisposition of the patient (Vlachopoulos et al. 2014).

A number of studies reported a statistically significant increase of cfPWV in patients with RA in comparison to their healthy counter partners (Mäki-Petäjä et al. 2006; Kocabay et al. 2012; Turkyilmaz et al. 2013). However, some other studies came to the opposite conclusion (Stamatelopoulos et al. 2009; Arida et al. 2015). Moreover, the association between aortic stiffness and different RA associated activity and chronicity markers is still somewhat unclear. Aim of this study was therefore to test the hypothesis of increased aortic stiffness in a group of RA patients in comparison to healthy subjects and to examine whether there is a correlation of cfPWV with various clinical and laboratory RA associated parameters.

\section{Methods}

We analyzed retrospectively routine measurements of carotid-femoral pulse wave velocity in 38 patients with RA and 25 subjects without systemic rheumatic or CV diseases. The measurements were conducted in our inpatient rheumatology clinic in Bad Kreuznach, Germany as a part of the diagnostic process. Patients with malignancy, pregnancy, age $<18$ years, active infection and $C V$ disease were excluded from the analysis.

All patients with RA met the 2010 American Rheumatism Association (ACR)/European League against Rheumatism (EULAR) classification criteria for RA.

\section{Arterial stiffness measurements}

The examination protocol of cfPWV was in accordance with the recommendations of the expert consensus document on arterial stiffness (Laurent et al. 2006). cfPWV was measured as the velocity value calculated through the distance between the carotid and femoral artery in meters $(\mathrm{m})$ divided by the time that one pulse wave needs to cover this distance in seconds $(\Delta \mathrm{s} / \Delta \mathrm{t})(\mathrm{m} / \mathrm{s})$. All of the measures were conducted from the author and two experienced independent medical assistants using a validated non-invasive device (Vicorder ${ }^{\circledR}$, SMT medical $\mathrm{GmbH} \& \mathrm{Co}$ ).

cfPWV was assessed three consecutive times in every patient and the average value of the measures was documented and used in the statistical analysis.

During the procedure, a neck pad was placed around the neck of the patient. The pad contained a small bladder which was placed over the carotid artery. A cuff (similar to blood pressure cuffs) was then strapped at the thigh of the patient. The bladder of the neck pad and the cuff inflated as the test started. After deflation pressure waves from the carotid and the femoral artery appeared on the screen of a connected laptop. The waves were then recorded simultaneously and the time delay between carotid and femoral wave was determined.

\section{Data collection}

In addition to epidemiological data of both groups (gender, age), clinical and laboratory parameters of chronicity and activity in the RA group such as disease duration, disease activity score 28 (DAS28) as well as its components [C-reactive protein (CRP), count of tender (TJC) and swollen joints (SJC), visual analogue scale (VAS)] were examined. Values of ANA, rheumatoid factor and anti-CCP-antibodies were also recorded. Finally, the presence of traditional $\mathrm{CV}$ risk factors such as smoking, known arterial hypertension, obesity [Body Mass Index (BMI) $\left.>25 \mathrm{~kg} / \mathrm{m}^{2}\right]$, hyperlipidemia and type II diabetes of the subjects of both groups were documented.

\section{Statistical analysis}

The assumption of normality of distribution was evaluated through the Shapiro-Wilk test. Continuous variables were found to be skewed and therefore presented as median (25th and 75th percentiles). Categorical variables were summarized as absolute (n) and relative (\%) frequencies.

The difference of cfPWV values between RA patients and controls was evaluated through Mann-Witney U test as cfPWV was not normally distributed. This test was also used to evaluate the association between cfPWV and categorical variables with two categories. In order to assess the correlation between cfPWV and continues characteristics, the Spearman correlation coefficient was used.

Furthermore, difference of cfPWV values between RA patients and controls after controlling for possible confounding factors was examined through an appropriate multivariate model. The same statistical model was used to check for the effect of confounding factors on the established correlations among patients with RA.

Descriptive statistics, regression analyses and tests have only been analysed by referring to cases without missing values. A probability value of 0.05 was considered statistically significant. We made no formal adjustment for the number of performed tests. Thus, the p-values should be considered to be exploratory. All statistical calculations were performed using the SPSS version 22.0 software (SPSS Inc, Chicago, Il, USA).

\section{Results}

There was a similar distribution between patients with RA and controls regarding gender, arterial hypertension, obesity, diabetes mellitus and hyperlipidemia (Table 1). 
Patients with RA had a higher average age in comparison to the control individuals $(p=0.027)$ and the RA-group included more smokers than the control group (34.2 vs. $12 \%, p=0.041$ ) (Table 1 ).

\section{Association between group (RA vs. control) and cfPWV} cfPWV average was found to be statistically significantly higher in the RA group compared to control group [8.75 (7.90-9.85) vs 8.10 (7.25-8.65) $\mathrm{m} / \mathrm{s}, p=0.015$ ] (Fig. 1).

To adjust for factors which had a statistically different distribution in the control and RA group (age and nicotine use), and therefore could have a confounding effect on the results, multiple regressions were performed.

The age-adjusted statistical model revealed that cfPWV was only marginally higher in the RA group in comparison to the control group [ $-0.7295 \% \mathrm{CI}(-1.50$ to $0.04), p=0.062]$. Moreover, adjustment for both age and nicotine use revealed a non-significant statistical difference between cfPWV in the two groups $[-0.58795 \% \mathrm{CI}$ $(-1.38$ to 0.201$), p=0.144]$.

Table 1 Descriptive characteristics by group

\begin{tabular}{|c|c|c|c|}
\hline & $\begin{array}{l}\text { Control } \\
(n=25)\end{array}$ & RA $(n=38)$ & $\begin{array}{l}\text { Significance } \\
(p)\end{array}$ \\
\hline Age (years) ${ }^{\mathrm{a}}$ & $56(42,59.5)$ & $61(50.50-70.25)$ & $0.027^{*}$ \\
\hline Gender (female) & $21(84.0 \%)$ & $32(84.2 \%)$ & 0.982 \\
\hline $\begin{array}{l}\text { Nicotine use } \\
\text { (smokers) }\end{array}$ & $3(12.0 \%)$ & $13(34.2 \%)$ & $0.041^{*}$ \\
\hline $\begin{array}{l}\text { Arterial hyperten- } \\
\text { sion }\end{array}$ & 12 (48.0\%) & $18(47.4 \%)$ & 0.960 \\
\hline $\begin{array}{l}\text { Obesity } \\
\qquad\left(\mathrm{BMl}>25 \mathrm{~kg} / \mathrm{m}^{2}\right)\end{array}$ & $11(44.0 \%)$ & $19(50 \%)$ & 0.500 \\
\hline Hyperlipidemia & $5(20.0 \%)$ & $12(31.6 \%)$ & 0.250 \\
\hline $\begin{array}{l}\text { Diabetes mellitus } \\
\text { (type II) }\end{array}$ & $1(4.0 \%)$ & $5(13.2 \%)$ & 0.214 \\
\hline $\begin{array}{l}\text { ANA positivity } \\
(>1: 80)\end{array}$ & - & $5(13.9 \%)$ & - \\
\hline $\operatorname{BSG}(\mathrm{mm} / \mathrm{h})^{\mathrm{a}}$ & - & $25(14.00-55.50)$ & - \\
\hline $\operatorname{VAS}(\mathrm{mm})^{\mathrm{a}}$ & - & $7(5.75-8.00)$ & - \\
\hline $\mathrm{CRP}(\mathrm{mg} / \mathrm{dL})^{\mathrm{a}}$ & - & $1.1(0.36-2.62)$ & - \\
\hline DAS $28(B S G)^{a}$ & - & $5.10(4.10-5.75)$ & - \\
\hline DAS $28(C R P)^{a}$ & - & $4.50(3.45-4.92)$ & - \\
\hline $\begin{array}{l}\text { Disease duration } \\
\text { (years) }^{\mathrm{a}}\end{array}$ & - & $11(4.00-17.00)$ & - \\
\hline $\mathrm{RF}(\mathrm{IU} / \mathrm{ml})^{\mathrm{a}}$ & - & $103(6.55-322.50)$ & - \\
\hline $\operatorname{CCP}(I \mathrm{U} / \mathrm{ml})^{\mathrm{a}}$ & - & $117(7.25-340.00)$ & - \\
\hline $\mathrm{TJC}^{\mathrm{a}}$ & - & $4.50(1.00-7.75)$ & - \\
\hline $\mathrm{SJC}^{\mathrm{a}}$ & - & $2(0.00-4.00)$ & - \\
\hline
\end{tabular}

The rest of data are presented as absolute $(\mathrm{n})$ and relative frequency (\%) $R A$ rheumatoid arthritis, $A N A$ antinuclear antibodies, $R F$ rheumatoid factor, Anti$C C P$ cyclic citrullinated peptide antibodies, DAS28 disease activity score $28, C R P$ C-reactive protein, $T J C$ tender joints count, $S J C$ swollen joints count

* $p<0.05$

a Data are presented as median (inter quartile range) as they are not normally distributed

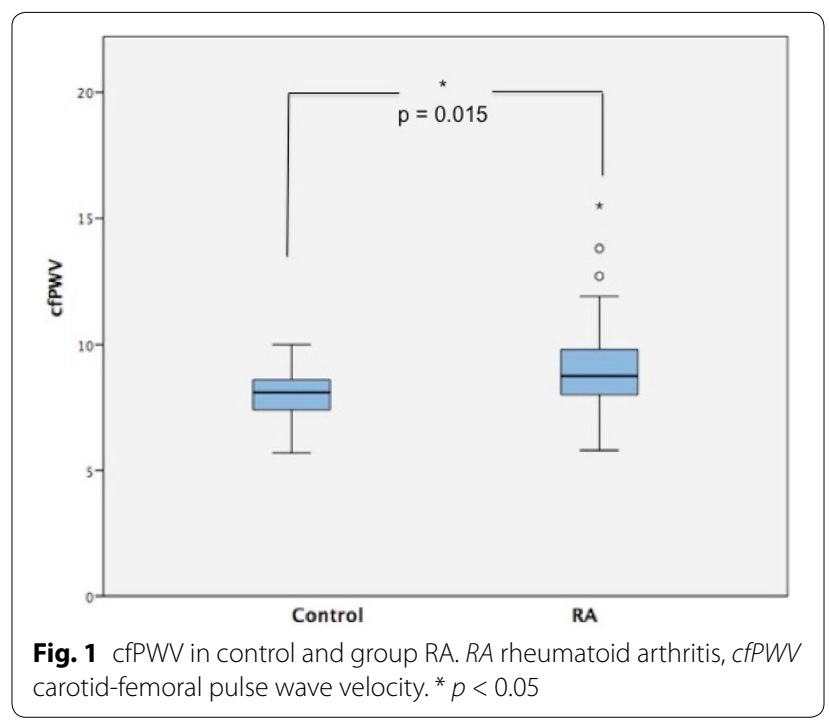

\section{Associations of cfPWV within RA-group}

Among patients with RA, it was found that cfPWV is significantly correlated with patients' age and RF. This means that older patients have higher values of cfPWV (rho $=0.544, p=0.001$ ) (Table 2; Fig. 2) and higher RF levels are associated with higher cfPWV values (rho $=0.369, p=0.025$ ) (Table 2). Moreover, cfPWV was found to be marginally positively correlated with TJC (rho $=0.330, p=0.065)$ and obesity $[8.80(8.50-10.30)$ vs. $8.60(7.2-9.15) \mathrm{m} / \mathrm{s}, p=0.071]$ (Tables 2,3$)$.

However, after adjusting for possible confounders (age, obesity) the association between RF and cfPWV did not

Table 2 Association between quantitative patients' characteristics and cfPWV in RA patients

\begin{tabular}{lcl}
\hline & Rho & Significance $(\boldsymbol{p})$ \\
\hline Age & 0.544 & $0.001^{*}(-)$ \\
Disease duration & 0.021 & $0.903(0.913)^{\ddagger}$ \\
CRP & -0.027 & $0.876(0.053)^{\ddagger}$ \\
ESR & -0.031 & $0.859(0.170)^{\ddagger}$ \\
RF & 0.369 & $0.025^{*}(0.462)^{\ddagger}$ \\
Anti-CCP & 0.142 & $0.401(0.345)^{\ddagger}$ \\
TJC & 0.330 & $0.065\left(0.034^{*}\right)^{\ddagger}$ \\
SJC & -0.065 & $0.713(0.744)^{\ddagger}$ \\
VAS & 0.117 & $0.509(0.763)^{\ddagger}$ \\
DAS28 (ESR) & 0.191 & $0.331(0.658)^{\ddagger}$ \\
DAS28 (CRP) & 0.213 & $0.266(0.499)^{\ddagger}$
\end{tabular}

Spearman rho was used

$R A$ rheumatoid arthritis, $C R P C$-reactive protein, ESR erythrocyte sedimentation rate, $R F$ rheumatoid factor; Anti-CCP cyclic citrullinated peptide antibodies, $T J C$ tender joints count, $S J C$ swollen joints count, VAS visual analogue scale, DAS28 disease activity score 28

${ }^{*} p<0.05$

* in parentheses $p$ values adjusted for age and obesity by multiple regression 


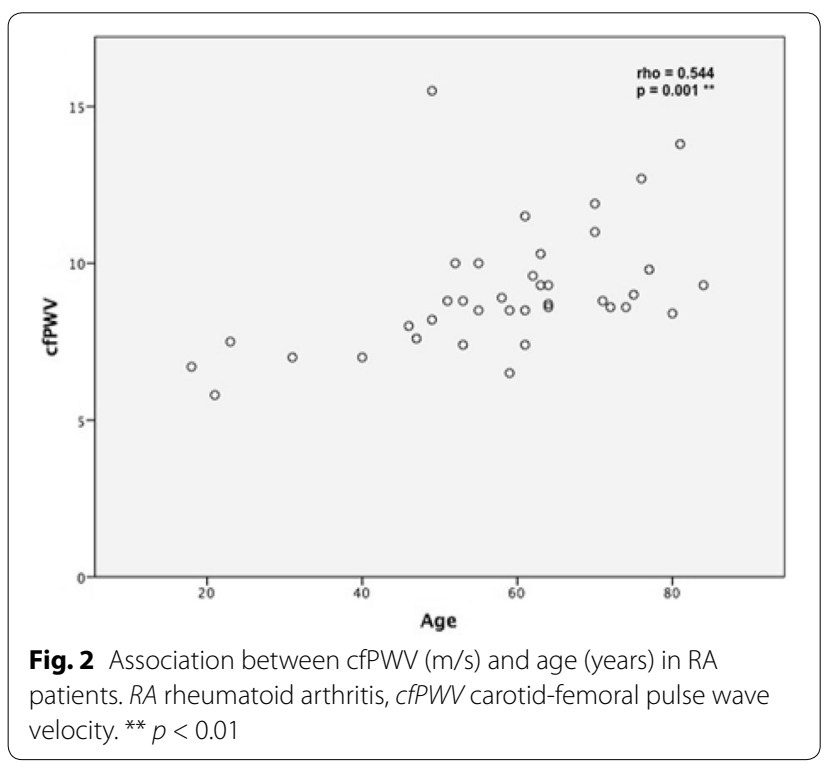

Table 3 Associations between qualitative patients' characteristics and cfPWV $(\mathrm{m} / \mathrm{s})$ in RA patients

\begin{tabular}{lll}
\hline & $\begin{array}{l}\text { Median } \\
(\mathbf{2 5 - 7 5} \text { percentiles) }\end{array}$ & Significance (p) \\
\hline $\begin{array}{ll}\text { Gender } \\
\text { Male }\end{array}$ & $8.55(8.20-8.93)$ & $0.471(0.598)^{\ddagger}$ \\
Female & $8.80(7.63-10.00)$ & \\
ANA & & \\
Positive (>1:80) & $8.70(8.00-9.60)$ & $0.993(0.768)^{\ddagger}$ \\
Negative & $8.80(7.10-10.50)$ & \\
Nicotine & & \\
Non-smokers & $8.70(7.66-9.75)$ & $0.937(0.617)^{\ddagger}$ \\
Smokers & $8.70(8.05-9.45)$ & \\
Arterial hypertension & & \\
No & $8.60(7.00-9.30)$ & $0.176(0.059)^{\ddagger}$ \\
Yes & $8.75(8.50-10.00)$ & \\
Obesity (BMI > 25 kg/m²) & & \\
Non-obese & $8.60(7.20-9.15)$ & $0.071(-)^{\ddagger}$ \\
Obese & $8.80(8.50-10.30)$ & \\
Hyperlipidemia & & $0.867(0.424)^{\ddagger}$ \\
No & $8.75(8.05-9.30)$ & \\
Yes & $8.90(7.45-10.23)$ & \\
Diabetes mellitus (type II) & $8.60(7.63-9.53)$ & \\
No & $9.30(8.20-12.90)$ & \\
Yes & & \\
\hline
\end{tabular}

Mann-Witney $U$ test was used

$R A$ rheumatoid arthritis, CfPWV carotid-femoral pulse wave velocity, ANA antinuclear antibodies, $B M /$ body mass index

₹ in parentheses $\mathrm{p}$ values adjusted for age and obesity by multiple regression remain statistically significant $(p=0.462)$ (Table 2$)$. On the contrary, the statistical significance of the association between cfPWV and TJC increased [0.051 $95 \% \mathrm{CI}$ (0.008-0.207), $p=0.034$ ] (Table 2).

Control of all results through adjustment for the same confounding factors showed marginal correlations of cfPWV with CRP [0.105 $95 \%$ CI $(-0.410$ to $0.003), p=0.053]$ and arterial hypertension [0.566 $95 \%$ CI ( -0.039 to 2.179$), p=0.059$ ]. Finally, gender, ANA positivity, nicotine use, hyperlipidemia, diabetes, disease duration, ESR, anti-CCP, SJC, VAS, DAS28 (ESR) and DAS28 (CRP) did not significantly correlate with cfPWV in the adjusted statistical models (all $p>0.05$, Tables 2,3 ).

\section{Conclusions}

\section{Pain and aortic stiffness}

The novel information of this exploration is the determined association between cfPWV and TJC in patients with RA. To our knowledge, there are no published studies in which the relationship between aortic stiffness and isolated parameters of the DAS28 in RA patients were investigated. Interestingly enough, cfPWV did not correlate with other parameters of disease activity such as SJC, VAS, ESR. The association between CRP and cfPWV was statistically marginal.

These results might reveal a closer association of cfPWV with joint tenderness and pain than with the acute joint-located inflammation in the course of arthritis.

It has been postulated that patients with RA show an increased sensibility to noxious stimulation (hyperalgesia) at both disease-affected and-non-affected regions (Wendler et al. 2001; Leffler et al. 2002; Edwards et al. 2009). RA is characterized from an altered pain processing in the central nervous system, which is suggested through the typical for this disease autonomic dysfunction and bilateral involvement (Edwards et al. 2009). Additionally, peripheral sensitization of primary afferent nociceptive neurons in inflamed sites plays an important role in the development of pain symptoms. Responsible for this sensitization are various proinflammatory and nociceptive molecules such as cytokines, prostaglandin, neuropeptides and bradykinin. These components are locally produced through inflamed tissue and initiate the process of pain transmission (Sprott 2008).

On the other hand, acute pain has been linked to hyperactivity of the sympathetic nervous system (SNS) (Pickering 2003). Particularly, pain stimuli can lead to a significant increase of both muscle sympathetic nerve activity (MSNA) and blood pressure (Fagius et al. 1989; 
Nordin and Fagius 1995). However, MSNA has been shown to associate independently of blood pressure with aortic stiffness in healthy individuals (Swierblewska et al. 2010). SNS-activation might promote arterial wall changes (fibrosis, growth of vascular muscles), which lead to an increase of arterial stiffness (Swierblewska et al. 2010). Moreover, SNS can cause an increase of aortic stiffness through its interaction with the renin-angiotensin-aldosterone system (Mancia et al. 2006). Finally, SNS-hyperactivity has been linked to endothelial dysfunction (Sverrisdóttir et al. 2010), which although distinct in terms of pathophysiology, cannot be completely separated from arterial stiffness (Nigam et al. 2003).

Summarizing, there might be a pathophysiological link between pain and the development of aortic stiffness through the effects of increased MSNA on the aortic vasculature.

Until now, the relationship between aortic stiffness and pain has been examined in studies which however included patients without the burden of a systemic inflammatory rheumatic disease (Lee et al. 2011; Kim et al. 2010; Vizzardi et al. 2014; Jin et al. 2015). Interestingly, female patients with fibromyalgia syndrome had statistically higher values of brachial ankle pulse wave velocity (baPWV, a marker of peripheral arterial stiffness) in comparison to their healthy counter partners (Lee et al. 2011). The authors implied a catecholamine associated activation of the autonomic nervous system, which led to an increase of arterial stiffness through endothelial impairment (Wittstein et al. 2005). The same marker was also found to be increased in patients with lumbar disc herniation (Jin et al. 2015). According to the authors, one of the main reasons for the increase of arterial stiffness in those patients was reduced physical activity.

Furthermore, increased augmentation index (indirect marker of arterial stiffness) and baPWV as well as endothelial dysfunction have been reported in patients with migraines (Jiménez Caballero and Muñoz Escudero 2013; Ikeda et al. 2011). Nevertheless, in both studies the increase of arterial stiffness was not linked to pain and its pathophysiological effects. Particularly, in the article of Jiménez Caballero and Muñoz Escudero (2013) the qualitative reduction and loss of function of endothelial progenitor cells, was among others discussed as a cause of impaired endothelial function in patients with migraine (Lee et al. 2008).

\section{RA-associated parameters and aortic stiffness}

Our analysis showed higher mean cfPWV values in the RA group in comparison to the control group. The results however, did not remain statistically significant after controlling for possible confounding factors. Other researchers found cfPWV to be increased in patients with RA in comparison to healthy individuals (MäkiPetäjä et al. 2006; Kocabay et al. 2012; Turkyilmaz et al. 2013). On the other hand, cfPWV was not found to be statistically different in a population of RA patients who were free of $\mathrm{CV}$ risk factors when compared to a group of age and gender matched healthy controls (Arida et al. 2015). Furthermore, in another study cfPWV was found to be only statistically marginally increased in patients with RA in comparison to controls after adjustment for confounding factors (Stamatelopoulos et al. 2009). These conflicting data could be due to heterogeneity of study protocols regarding the amount, age and CV risk factors of examined RA patients and control subjects. A further explanation could be provided through differences in administered immunosuppressant therapies throughout the years, since new RA directed biological drugs lead to a reduction of arterial stiffness (Mäki-Petäjä et al. 2006; Galarraga et al. 2009; Protogerou et al. 2011; Vassilopoulos et al. 2015).

Our exploration did not reveal statistically significant correlations between cfPWV and anti-CCP-antibodies, RF, ANA or disease duration. The presence of anti-CCP-antibodies has been shown to correlate with subclinical vasculopathy (Szekanecz et al. 2007). AntiCCP-positive-patients show an increase of carotid intima media thickness (cIMT), which is as a marker of subclinical arteriosclerosis (Gerli et al. 2007; Arnab et al. 2013). Furthermore, positivity for anti-CCP is described to be a risk factor for the occurrence of an ischemic cardiac disease (Arnab et al. 2013). Even though anti-CCP-positive patients possess a higher $\mathrm{CV}$ risk, there are, to our knowledge, no data to support a direct correlation of this antibody with cfPWV (Pieringer et al. 2010; Provan et al. 2011). The presence of RF has been shown to associate with higher cIMT values and endothelial dysfunction in patients with RA (Rojas-Villarraga et al. 2008; Sahari et al. 2014), but not directly with aortic stiffness (Mäki-Petäjä et al. 2006). We are not aware of studies who examined the relationship between ANA and cfPWV. Finally, our findings regarding the missing association between aortic stiffness and RA disease duration are in agreement with other RA/cfPWV studies (Mäki-Petäjä et al. 2006; Provan et al. 2011; Li et al. 2013).

The present exploration has some limitations: First of all, the low number of patients and control subjects may limit the validity of the results. However, a lot of published cFPWV studies included similar amount of subjects. Secondly, the presence of cardiovascular risk factors in both groups may have had a confounding effect on the results. Despite the fact that adjusted statistical analyses for some of the possible confounders were conducted, the outcomes of the study should be interpreted cautiously. 
In conclusion, we report for the first time that aortic stiffness can correlate with the count of tender joints in patients with RA. The association between joint tenderness and aortic stiffness, if proven, could influence future treatment decisions in clinical practice. More studies are needed, in order to verify this finding and to fully understand the mechanisms behind it.

\section{Authors' contributions}

KT designed the study, gathered data, performed a part of the cfPWV measurements and wrote the manuscript. $\mathrm{MdB}$ and $\mathrm{IH}$ were involved in data analysis and statistical exploration. TT helped to draft the manuscript. PD revised the manuscript critically. AS conceived of the study, participated in its design and revised the manuscript critically. All authors read and approved the final manuscript.

\section{Author details \\ ${ }^{1}$ ACURA Rheumatology Clinics, Kaiser-Wilhelm-Str. 9-11, 55543 Bad Kreuznach, Germany. ${ }^{2}$ Institute of Medical Biostatistics, Epidemiology and Informatics (IMBEI), Johannes Gutenberg University, Mainz, Germany. ${ }^{3}$ First Department of Medicine, Johannes Gutenberg University, Mainz, Germany. ${ }^{4}$ Department of Orthopaedics and Trauma Surgery, Johannes Gutenberg University, Mainz, Germany,}

\section{Acknowledgements}

Special thanks to the medical assistants Mrs. Nicole Dirvonskis and Mrs. Petra Woog of the ACURA Rheumatology Clinic in Bad Kreuznach, Germany.

\section{Competing interests}

The authors declare that they have no competing interests.

Received: 16 February 2016 Accepted: 29 March 2016

Published online: 11 April 2016

\section{References}

Abou-Raya A, Abou-Raya S (2006) Inflammation: a pivotal link between autoimmune diseases and atherosclerosis. Autoimmun Rev 5:331-337

Arida A, Zampeli E, Konstantonis G et al (2015) Rheumatoid arthritis is sufficient to cause atheromatosis but not arterial stiffness or hypertrophy in the absence of classical cardiovascular risk factors. Clin Rheumatol 34:853-859

Arnab B, Biswadip G, Arindam P et al (2013) Anti-CCP antibody in patients with established rheumatoid arthritis: Does it predict adverse cardiovascular profile? J Cardiovasc Dis Res 4:102-106

Baulmann J, Nürnberger J, Slany J et al (2010) Arterial stiffness and pulse wave analysis. J Cardiovasc Pharmacol 135(Suppl):33-37

Del Rincón ID, Williams K, Stern MP et al (2001) High incidence of cardiovascular events in a rheumatoid arthritis cohort not explained by traditional cardiac risk factors. Arthritis Rheum 44:2737-2745

Edwards RR, Wasan AD, Bingham CO et al (2009) Enhanced reactivity to pain in patients with rheumatoid arthritis. Arthritis Res Ther 11:R61

Fagius J, Karhuvaara S, Sundlöf G (1989) The cold pressor test: effects on sympathetic nerve activity in human muscle and skin nerve fascicles. Acta Physiol Scand 137:325-334

Galarraga B, Khan F, Kumar P et al (2009) Etanercept improves inflammation-associated arterial stiffness in rheumatoid arthritis. Rheumatology 48:1418-1423

Gerli R, Sherer Y, Bocci EB et al (2007) Precocious atherosclerosis in rheumatoid arthritis: role of traditional and disease-related cardiovascular risk factors. Ann N Y Acad Sci 1108:372-381

Ikeda K, Hirayama T, Iwamoto Ket al (2011) Pulse wave velocity study in middle-aged migraineurs at low cardiovascular disease risk. Headache 51:1239-1244

Jiménez Caballero PE, Muñoz Escudero F (2013) Peripheral endothelial function and arterial stiffness in patients with chronic migraine: a case-control study. J Headache Pain 14:8
Jin G, Cao ZG, Zhang YN, Li YSB (2015) Physical activity is associated with elevated arterial stiffness in patients with lumbar disc herniation. J Spinal Disord Tech 28:E30-E34

Kim SK, Kim KS, Lee YS, Park SHCJ (2010) Arterial stiffness and proinflammatory cytokines in fibromyalgia syndrome. Clin Exp Rheumatol 28:71-77

Kocabay G, Hasdemir H, Yildiz M (2012) Evaluation of pulse wave velocity in systemic lupus erythematosus, rheumatoid arthritis and Behcet's disease. J Cardiol 59:72-77

Laurent S, Cockcroft J, Van Bortel L et al (2006) Expert consensus document on arterial stiffness: methodological issues and clinical applications. Eur Hear J 27:2588-2605

Lee S-T, Chu K, Jung K-H et al (2008) Decreased number and function of endothelial progenitor cells in patients with migraine. Neurology 70:1510-1517

Lee JH, Cho Kl, Kim SM et al (2011) Arterial stiffness in female patients with fibromyalgia and its relationship to chronic emotional and physical stress. Korean Circ J 41:596-602

Leffler AS, Kosek E, Lerndal T et al (2002) Somatosensory perception and function of diffuse noxious inhibitory controls (DNIC) in patients suffering from rheumatoid arthritis. Eur J Pain 6:161-176

Li P, Han C-X, Ma C-L et al (2013) Determinants of brachial-ankle pulse wave velocity in Chinese patients with rheumatoid arthritis. Clin Dev Immunol 2013:342869

Mäki-Petäjä KM, Hall FC, Booth AD et al (2006) Rheumatoid arthritis is associated with increased aortic pulse-wave velocity, which is reduced by antitumor necrosis factor-alpha therapy. Circulation 114:1185-1192

Mancia G, Dell'Oro R, Quarti-Trevano F et al (2006) Angiotensin-sympathetic system interactions in cardiovascular and metabolic disease. J Hypertens Suppl 24:S51-S56

Nigam A, Mitchell G, Lambert J, Tardif J (2003) Relation between conduit vessel stiffness (assessed by tonometry) and endothelial function (assessed by flow-mediated dilatation) in patients with and without coronary heart disease. Am J Cardiol 92:395-399

Nordin M, Fagius J (1995) Effect of noxious stimulation on sympathetic vasoconstrictor outflow to human muscles. J Physiol 489(Pt 3):885-894

Pickering T (2003) Effects of stress and behavioral interventions in hypertension. Pain and blood pressure. J Clin Hypertens 5:359-361

Pieringer H, Stuby U, Pohanka E, Biesenbach G (2010) Arterial stiffness in a muscular artery in women with longstanding rheumatoid arthritis compared with healthy controls and patients with traditional cardiovascular risk factors. Rheumatol Int 30:1335-1339

Protogerou AD, Zampeli E, Fragiadaki K et al (2011) A pilot study of endothelial dysfunction and aortic stiffness after interleukin-6 receptor inhibition in rheumatoid arthritis. Atherosclerosis 219:734-736

Provan S, Semb A, Hisdal J (2011) Remission is the goal for cardiovascular risk management in patients with rheumatoid arthritis: a cross-sectional comparative study. Ann Rheum Dis 70:812-817

Rojas-Villarraga A, Ortega-Hernandez O-D, Gomez LF et al (2008) Risk factors associated with different stages of atherosclerosis in Colombian patients with rheumatoid arthritis. Semin Arthritis Rheum 38:71-82

Sahari N, Shaharir S, Ismail M et al (2014) Subclinical atherosclerosis among rheumatoid arthritis patients without overt cardiovascular risk factors. Mod Rheumatol 24:920-925

Sprott H (2008) Peripheral mechanisms of joint pain with special focus on the synovial fibroblast. Z Rheumatol 67:640-645

Stamatelopoulos KS, Kitas GD, Papamichael CM et al (2009) Atherosclerosis in rheumatoid arthritis versus diabetes: a comparative study. Arterioscler Thromb Vasc Biol 29:1702-1708

Sverrisdóttir YB, Jansson LM, Hägg U, Gan L-M (2010) Muscle sympathetic nerve activity is related to a surrogate marker of endothelial function in healthy individuals. PLoS One 5:e9257

Swierblewska E, Hering D, Kara T et al (2010) An independent relationship between muscle sympathetic nerve activity and pulse wave velocity in normal humans. J Hypertens 28:979-984

Szekanecz Z, Kerekes G, Dér H et al (2007) Accelerated atherosclerosis in rheumatoid arthritis. Ann NY Acad Sci 1108:349-358

Turkyilmaz AK, Devrimsel G, Kirbas A et al (2013) Relationship between pulse wave velocity and serum YKL-40 level in patients with early rheumatoid arthritis. Rheumatol Int 33:2751-2756 
Vassilopoulos D, Gravos A, Vlachopoulos C et al (2015) Adalimumab decreases aortic stiffness independently of its effect in disease activity in patients with rheumatoid arthritis. Clin Rheumatol 34:359-364

Vizzardi E, Trichaki E, Bonadei I, Sciatti E, Salghetti F, Raddino RMM (2014) Elastic aortic properties in patients with X syndrome. Hear Lung Circ 23:114-118

Vlachopoulos C, Aznaouridis K, Stefanadis C (2014) Aortic stiffness for cardiovascular risk prediction: just measure it, just do it! J Am Coll Cardiol 63:647-649
Wendler J, Hummel T, Reissinger M et al (2001) Patients with rheumatoid arthritis adapt differently to repetitive painful stimuli compared to healthy controls. J Clin Neurosci 8:272-277

Wittstein IS, Thiemann DR, Lima JAC et al (2005) Neurohumoral features of myocardial stunning due to sudden emotional stress. N Engl I Med 352:539-548

\section{Submit your manuscript to a SpringerOpen ${ }^{\circ}$ journal and benefit from:}

- Convenient online submission

- Rigorous peer review

- Immediate publication on acceptance

- Open access: articles freely available online

- High visibility within the field

- Retaining the copyright to your article

Submit your next manuscript at $\gg$ springeropen.com 